\title{
Risk Factors for Early Hemorrhagic Complications after Endovascular Coiling of Ruptured Intracranial Aneurysms
}

\author{
T. Ohta, K. Murao, K. Miyake, K. Takemoto, and K. Nakazawa
}

\begin{abstract}
BACKGROUND AND PURPOSE: The risk factors of early hemorrhagic complications after endovascular coiling are not well-known. We identified the factors affecting early hemorrhagic complications, defined as any expansion or appearance of hemorrhage shown by head CT in the initial 48 hours after coiling.
\end{abstract}

MATERIALS AND METHODS: We retrospectively reviewed a series of 93 patients who underwent coiling for a ruptured saccular aneurysm between 2006 and 2012 at our hospital.

RESULTS: Five patients showed early hemorrhagic complications, and all involved an expansion of the existing intracerebral hematoma immediately after coiling. The associated risk factors were accompanying intracerebral hemorrhage at onset $(P<.001)$, postoperative antiplatelet therapy $(P<.001)$, and thromboembolic complications $(P=.044)$. In the accompanying intracerebral hemorrhage group, the associated risk factors were postoperative antiplatelet therapy $(P=.044)$ and earlier initiation of coiling (9.8 \pm 6.5 versus $28.1 \pm 24.0$ hours, $P=.023$ ). Early hemorrhagic complications were significant risk factors for worse clinical outcome (modified Rankin Scale, $2.02 \pm 2.21$ versus $4.4 \pm 2.30, P=.022)$. None of the 93 patients showed further hemorrhage after the initial 48 hours after coiling.

CONCLUSIONS: The accompanying intracerebral hemorrhage at onset, thromboembolic complications, postoperative antiplatelet therapy, and earlier initiation of coiling were the risk factors for early hemorrhagic complications.

ABBREVIATION: $\mathrm{ICH}=$ intracerebral hematoma

A recent guideline stated that endovascular coiling should be considered if ruptured aneurysms were judged to be technically amenable to both endovascular coiling and neurosurgical clipping. ${ }^{1}$ Consequently, the use of coiling for ruptured saccular cerebral aneurysms has been increasing. One problem is that the incidence of periprocedural hemorrhagic complications within 30 days was reportedly high in the endovascular arm of a study, ${ }^{2}$ and they were reported to be associated with high mortality and morbidity. ${ }^{3}$ The risk factors for such early hemorrhagic complications are not well-known. Although the word "rebleeding" is often used for perioperative hemorrhagic complications, it is also unclear whether "rebleeding" is really caused by a rerupture of the aneurysm or is due to other mechanisms. Therefore, we analyzed cases of coiling for ruptured saccular aneurysms in which early

Received March 9, 2014; accepted after revision May 19.

From the Department of Neuroendovascular Treatment, Shiroyama Hospital, Osaka, Japan.

Please address correspondence to Tsuyoshi Ohta, MD, Department of Neuroendovascular Treatment, Shiroyama Hospital, 2-8-1 Habikino, Habikino City, Osaka 583-0872, Japan; e-mail: tsuyoshi@ya2.so-net.ne.jp

http://dx.doi.org/10.3174/ajnr.A4033 hemorrhagic complications had occurred within 48 hours after coiling, to identify factors affecting these complications.

\section{MATERIALS AND METHODS}

We retrospectively reviewed a series of 93 patients who underwent coiling for a ruptured saccular aneurysm between September 2006 and March 2012 at our hospital. Only patients for whom coiling was performed within 3 days of diagnosis were included. We generally recommend early treatment for subarachnoid hemorrhage; however, 7 patients had unstable circulatory conditions that inhibited early interventions; 6 patients were first admitted to another hospital and then were referred to our hospital 1 or 2 days after onset. All patients presented with SAH confirmed by CT. Patients with accompanying intracerebral hematoma (ICH) other than SAH on preprocedural head CT were defined as having "accompanying ICH." The ruptured aneurysms were identified by CT angiography or digital subtraction angiography. The choice of clipping or coiling was determined by discussion with the neurosurgeons. If the patient had a large ICH with a widespread effect such as ICH with a poor World Federation of Neurosurgical Societies grade ${ }^{4}$ of IV and V, clipping with evac- 
Table 1: Characteristics and surgical outcome of the patients with early hemorrhagic complications

\begin{tabular}{ccccccc}
\hline $\begin{array}{c}\text { Age } \\
\text { (yr)/Sex }\end{array}$ & WFNS & ICH & $\begin{array}{c}\text { Intraoperative } \\
\text { Perforation }\end{array}$ & $\begin{array}{c}\text { Intraoperative } \\
\text { Thrombus Formation }\end{array}$ & $\begin{array}{c}\text { Postoperative } \\
\text { Antiplatelet Therapy }\end{array}$ & mRS \\
\hline $31 / F$ & IV & Yes & No & Yes & Yes & 1 \\
$81 / F$ & II & Yes & No & Yes & Yes & 6 \\
$57 / F$ & I & Yes & Yes & No & No & 3 \\
49/F & II & Yes & No & No & Yes & 6 \\
$62 / \mathrm{M}$ & V & Yes & No & Yes & \\
\hline
\end{tabular}

Note:-WFNS indicates World Federation Neurological Societies grade.

uation of the ICH was selected. If the patient had a small ICH with a minimal effect such as ICH with a good World Federation of Neurosurgical Societies grade, coiling was selected when technically feasible.

Coiling was started as soon as the operator, anesthesiologist, and the patient's medical condition were ready for the treatment. All treatments were performed with the patient under general anesthesia and having been given systemic heparinization with an activated clotting time of approximately 200-250 seconds. In cases with multiple aneurysms, the ruptured one was estimated by the distribution of the subarachnoid hemorrhage. Blood pressures were maintained within normal range. Coiling was performed simply or with an adjunctive technique such as balloon assist. No stents were applied. The strategy of coiling was to employ packing sufficient for hemostasis. Antiplatelet therapy was initiated when intraoperative thrombus formation was found. At the end of treatment, heparin was not reversed but was discontinued because the last activated clotting time was usually $<200 \mathrm{sec}-$ onds. Heparin was reversed with protamine if bleeding events occurred. General anesthesia was continued until the day after the procedure to avoid perioperative elevated intracranial pressure, which is associated with a patient's clinical outcome. ${ }^{5}$

The clinical condition before coiling was assessed according to the World Federation of Neurosurgical Societies grade. Early hemorrhagic complications were defined as an occurrence of new bleeding or enlargement of the pre-existing ICH within 48 hours after coiling. Early rebleeding from the treated aneurysm was assumed if postoperative CT showed an extravasation of contrast or the new hemorrhage was found only in the subarachnoid space. If the postoperative CT did not show any leakage of contrast and the new hemorrhage was added to $\mathrm{ICH}$, we defined the new hemorrhage as an expansion of the accompanying ICH. Diagnostic confirmation was accepted when CT showed an augmentation of any hemorrhage, including $\mathrm{SAH}$, intraventricular hematoma, or intracerebral hemorrhage, compared with that shown on the preprocedural CT, as judged by 2 physicians (T.O. and K.M.). Clinical outcomes were assessed according to the modified Rankin Scale on postoperative day $30 .^{6}$ The degree of aneurysmal occlusion was assessed by packing density calculated from the volume of the inserted coil divided by the preprocedural 3D aneurysmal volume, except for the intraoperative perforated cases because coil volume outside the aneurysm could not be evaluated in these cases.

\section{Statistical Analysis}

The Fisher exact test or Student $t$ test, as appropriate, was used to compare factors such as accompanying ICH, aneurysmal location, postoperative antiplatelet therapy, intraoperative perforation, and symptomatic thromboembolic complications, which were detected on postoperative head MR imaging; packing density (when calcu- lated); and time to coil in each group. $P$ values $<.05$ were considered statistically significant. The $P$ value was expressed to 2 significant figures; if the value was $<.001$, it was expressed as $P<.001$.

\section{RESULTS}

Coiling was performed in 93 patients, including 26 men and 67 women, $65.1 \pm$ 15.0 years of age. During the same period, clipping was performed in 136 patients. Multiple aneurysms were observed in 5 patients. The World Federation of Neurosurgical Societies grade was $2.80 \pm 1.54$, and the location of the aneurysms was supratentorial in 75 patients $(80.6 \%)$. The aneurysms were located in the anterior communicating artery (29 cases), internal carotid artery (17 cases), internal carotid-posterior communicating artery (15 cases), basilar tip and basilar-superior cerebellar artery (12 cases), middle cerebral artery ( 7 cases), vertebral artery-posterior inferior cerebellar artery and vertebrobasilar junction ( 4 cases), and other locations ( 9 cases). The maximum diameter of the aneurysms was $6.89 \pm 3.1$ mm. Eighteen patients (19.4\%) had accompanying ICH with SAH at the first head CT (accompanying ICH group). Significantly more patients with poor neurological status, as shown by World Federation of Neurosurgical Societies grades IV and V (66.7\% versus $29.3 \%$, respectively; $P=.006$ ) and poor clinical outcome, as shown by modified Rankin Scale grades 4 and 5 ( $66.7 \%$ versus $24 \%$, respectively; $P=.001$ ), were included in the accompanying $\mathrm{ICH}$ group than in the nonaccompanying $\mathrm{ICH}$ group.

Five patients had early hemorrhagic complications (5.4\%). All of the early hemorrhagic complications occurred within 24 hours. No patient had early hemorrhagic complications defined as $\mathrm{SAH}$ even in the 4 cases of intraoperative perforations. Hemorrhagic transformation of cerebral infarction was not observed. All early hemorrhagic complications involved the expansion of the accompanying ICH. Characteristics of the 5 patients, radiologic findings, and surgical outcomes are summarized in Table 1. Comparisons between the early hemorrhagic complications group and the nonhemorrhagic group are shown in Table 2. Accompanying ICH $(P<.001)$, postoperative antiplatelet therapy $(P<.001)$, and thromboembolic complications $(P=.044)$ were associated with early hemorrhagic complications. In 1 patient (case 3 ), endoscopic hematoma evacuation of the enlarged accompanying $\mathrm{ICH}$ was performed. Other cases were treated conservatively. Because all the early hemorrhagic complications presented as the expansion of the accompanying $\mathrm{ICH}$, a comparison within the accompanying $\mathrm{ICH}$ group was performed to analyze the risk factors for expansion. The associated risk factors for the enlargement of a pre-existing ICH were postoperative antiplatelet therapy $(P=$ $.044)$ and earlier initiation of coiling $(9.8 \pm 5.8$ versus $28.1 \pm 23.1$ hours, $P=.023$ ) (Table 3 ).

\section{Representative Case}

A 31-year-old woman presented with severe headache and disturbance of consciousness (World Federation of Neurosurgical Societies grade IV). Cerebral angiography revealed a left internal carotid-anterior choroidal artery bifurcation aneurysm with a maximum diameter of $4.5 \mathrm{~mm}$ (Fig $1 A$ ). With the patient under 
Table 2: Comparison of patient factors between the early hemorrhagic complications and no complications groups

\begin{tabular}{|c|c|c|c|}
\hline Factors & $\begin{array}{l}\text { Early Hemorrhagic } \\
\text { Group (5 Patients) }\end{array}$ & $\begin{array}{l}\text { Nonhemorrhagic } \\
\text { Group ( } 88 \text { Patients) }\end{array}$ & $P$ Value \\
\hline Accompanying intracerebral hematoma & $5(100 \%)$ & $13(14.8 \%)$ & $<.001^{\mathrm{a}}$ \\
\hline Supratentorial location & $5(100 \%)$ & $70(79.5 \%)$ & .58 \\
\hline Postoperative antiplatelet therapy & $3(60 \%)$ & $1(1.14 \%)$ & $<.001^{\mathrm{a}}$ \\
\hline Intraoperative perforation & $1(20 \%)$ & $4(4.55 \%)$ & .25 \\
\hline Thromboembolic complications & $2(40 \%)$ & $5(5.68 \%)$ & $.044^{\mathrm{a}}$ \\
\hline Packing attenuation (when calculated) & $26.6 \pm 3.7$ ( 3 cases $)$ & $29.4 \pm 8.2$ (83 cases) & .57 \\
\hline
\end{tabular}

Table 3: Risk factors for early hemorrhagic complications in the accompanying intracerebral hemorrhage group

\begin{tabular}{lccc}
\hline \multicolumn{1}{c}{ Factors } & $\begin{array}{c}\text { Early Hemorrhagic } \\
\text { Group (5 Patients) }\end{array}$ & $\begin{array}{c}\text { Nonhemorrhagic } \\
\text { Group (13 Patients) }\end{array}$ & \begin{tabular}{l}
$\boldsymbol{P}$ Value \\
\hline Supratentorial location
\end{tabular} \\
Postoperative antiplatelet therapy & 3 & 12 & .72 \\
Intraoperative perforation & 1 & 1 & $.044^{\mathrm{a}}$ \\
Time to coiling (hr) & $9.8 \pm 5.8$ & 0 & .28 \\
\hline
\end{tabular}

a Significant.
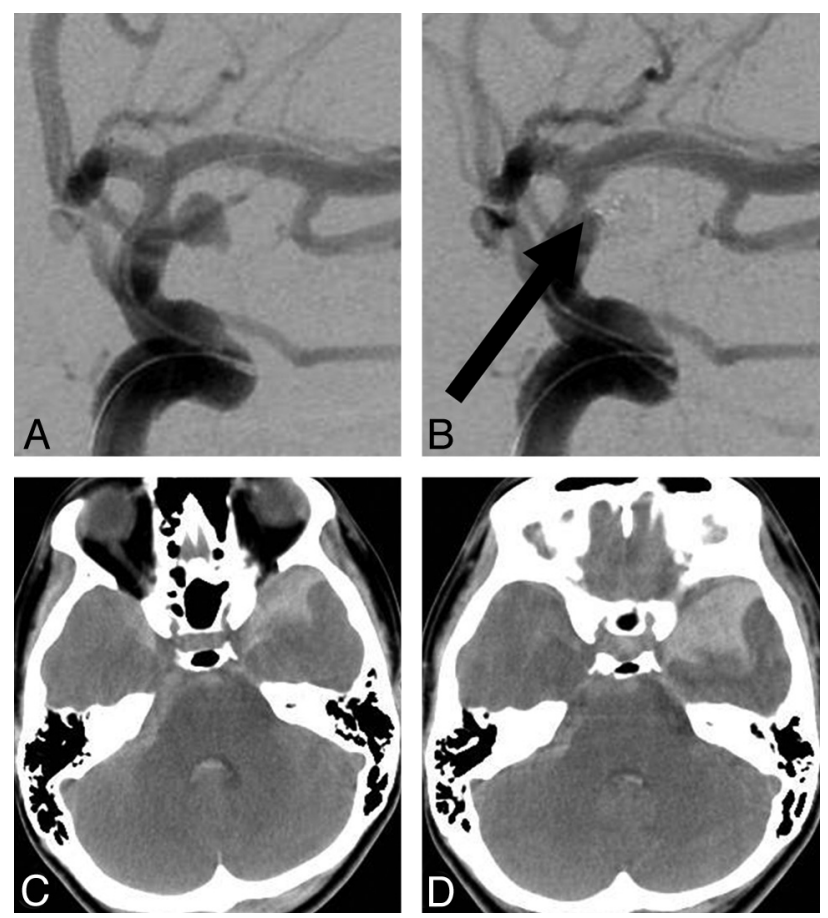

FIG 1. Representative case. Cerebral angiography reveals a left internal carotid-anterior choroidal artery bifurcation aneurysm $(A)$ and a small thrombus, indicated by the arrow after placement of coils $(B)$. Head CT on admission shows a subarachnoid hemorrhage with lobar hemorrhage (C). Head CT after the procedure does not show any leakage of contrast, but enlargement of the existing parenchymal temporal lobar hemorrhage is observed $(D)$.

systemic heparinization with injection of $4000 \mathrm{U}$ of heparin, coil embolization was performed by using the balloon-assist technique. After the placement of 4 bare platinum coils, we found a small thrombus (Fig 1B); after the administration of $100 \mathrm{mg}$ of aspirin and the addition of $1000 \mathrm{U}$ of heparin, the thrombus disappeared and 3 additional coils were added to achieve complete occlusion. Her preoperative head CT showed massive subarachnoid hemorrhage with lobar hemorrhage of the left temporal tip (Fig 1C). Postprocedural head CT showed enlargement of the existing left temporal lobar hemorrhage compared with the pre- operative CT (Fig 1D). Fortunately, she fully recovered with minor sequelae and resumed her previous activities (modified Rankin Scale 1).

\section{DISCUSSION}

The greatest risk of hemorrhagic complications associated with endovascular coil treatment of aneurysms is during the initial 48 hours. $^{7}$ Reducing early hemorrhagic complications may produce better clinical outcomes following coiling.

In our study, no patient showed early hemorrhagic complications defined as $\mathrm{SAH}$ even in the case of intraoperative perforations. All complications involved the expansion of the pre-existing hematomas. Some reports have stated that early hemorrhagic complications are possible even when the treatment of a patient appears to be successful on angiography. ${ }^{8}$ We speculated that early hemorrhagic complications may be caused by mechanisms other than arterial bleeding from the coiled ruptured aneurysm. The term "acute rebleeding" may limit the situations involving the rerupture of the aneurysm. Therefore, we referred to these cases as those involving "early hemorrhagic complications."

In our study, the presence of ICH and postoperative antiplatelet therapy were identified as risk factors. Jartti et al $^{9}$ evaluated 194 patients with SAH and found that early rehemorrhage appeared as an enlargement of the initial ICH in all these patients. Dmytriw et $\mathrm{al}^{10}$ reported a series of 137 cases with ruptured aneurysms that underwent endovascular treatment and 2 cases with early hemorrhagic complications (1.4\%) for which one received recombinant tissue plasminogen activator and both received acetylsalicylic acid. Egashira et $\mathrm{al}^{11}$ reported the results in the endovascular treatment of 101 consecutive cases with SAH and found that 14 (13.9\%) had hematoma growth unrelated to aneurysmal rebleeding. The other reported risk factors for early hemorrhagic complications after coiling are an occurrence of a residual aneurysm at the end of a coiling procedure, ${ }^{12}$ an intra-arterial infusion of abciximab or tirofiban, ${ }^{3}$ and maintenance of anticoagulation after the procedure. ${ }^{3}$ Antiplatelet therapy may inhibit the hemostasis process of the existing ICH. The presence of intraoperative thrombus formation was also a risk factor, which may be because of subsequent antiplatelet therapy. The incidence of early hemorrhagic complications has been reported as $1 \%$, even after angiographically successful coiling. ${ }^{8}$ In our study, there was no significant difference in packing density between the groups.

The presence of $\mathrm{ICH}$ is a predictor of unfavorable outcome of $\mathrm{SAH},{ }^{13}$ especially hematoma growth without aneurysmal rebleeding. ${ }^{11}$ As reported previously, ${ }^{11}$ ultra-early embolization performed within 6 hours of onset was a risk factor for early hemorrhagic complications. An earlier intervention led to an earlier initiation of anticoagulation therapy and may have promoted expansion of the existing ICH. Intentional delay in performing coiling may be considered, but because early aneurysmal rebleeding is associated with very poor outcomes, urgent prevention is recommended. ${ }^{1}$ Therefore, if treatment can be initiated earlier than 24 
hours in cases with accompanying ICH, clipping and evacuation of the hematoma may be a better approach. If coiling is selected, it should be performed without antiplatelet therapy, and clot evacuation should be performed subsequently to prevent the risk of early hemorrhagic complications. Endoscopic aspiration of hematomas was reported to improve outcomes ${ }^{14,15}$ and may be preferred over craniotomy, as in our single case.

Some researchers reported that intraprocedural perforations have appeared to be associated with relatively high rates of morbidity and mortality. ${ }^{16,17}$ Other researchers have reported that new neurologic deficits directly associated with aneurysm perforation were found in only $20 \%$ of cases with leakage of contrast. ${ }^{18}$ In our study, 4 intraoperative perforations occurred, but these were not a significant risk factor for either early hemorrhagic complications or worse clinical outcomes. If we found intraoperative perforation, we quickly reversed heparin and lowered the blood pressure. We never pulled back the protruded coil or microcatheter. We rapidly coiled from that point, and the perforated portion was closed in a few minutes. With this method, fortunately, we saw no increase in the incidence of $\mathrm{SAH}$. Aneurysm perforation may not necessarily be associated with worse clinical outcome.

Thromboembolic complications and postoperative antiplatelet therapy were identified as risk factors for early hemorrhagic complications. From a technical point of view, it is better to avoid overly aggressive coiling, which may lead to these complications. Antiplatelet agents and anticoagulants have been suggested as risk factors for the early expansion of spontaneous ICH. ${ }^{19,20}$ Even if apparent thromboembolic complications followed, postoperative antiplatelet therapy may be more hazardous in cases with accompanying ICH.

A limitation of this study was its retrospective nature, which made it impossible to determine whether the factors affecting early hemorrhagic complications were causes of or simply results associated with early complications after coiling. In addition, because we avoided coiling in patients with large ICHs with widespread effects, over- or underestimation of the importance of ICH as it relates to the risk of postcoiling hemorrhage may have occurred. However, our results may be helpful in determining the clinical steps that should be taken for patients who undergo coiling for ruptured aneurysms, particularly those with accompanying $\mathrm{ICH}$.

\section{CONCLUSIONS}

We analyzed early hemorrhagic complications after coiling to treat ruptured cerebral aneurysms. Accompanying ICH, thromboembolic complications, postoperative antiplatelet therapy, and earlier initiation of coiling were identified as risk factors. In patients for whom there is a high probability of early hemorrhagic complications, clipping and/or clot evacuation may be selected.

Disclosures: Tsuyoshi Ohta-UNRELATED: Payment for Lectures (including service on Speakers Bureaus): Kaneka, Stryker, Comments: paid for lectures on device training.

\section{REFERENCES}

1. Connolly E Jr, Rabinstein AA, Carhuapoma JR, et al. Guidelines for the management of aneurysmal subarachnoid hemorrhage: a guideline for healthcare professionals from the American Heart Association/American Stroke Association. Stroke 2012;43:1711-37

2. Molyneux A, Kerr R, Stratton I, et al. International Subarachnoid Aneurysm Trial (ISAT) of neurosurgical clipping versus endovascular coiling in 2143 patients with ruptured intracranial aneurysms: a randomised trial. Lancet 2002;360:1267-74

3. Cho YD, Lee JY, Seo JH, et al. Early recurrent hemorrhage after coil embolization in ruptured intracranial aneurysms. Neuroradiology 2012;54:719-26

4. Report of World Federation of Neurological Surgeons Committee on a Universal Subarachnoid Hemorrhage Grading Scale. J Neurosurg 1988;68:985-86

5. Heuer GG, Smith MJ, Elliott JP, et al. Relationship between intracranial pressure and other clinical variables in patients with aneurysmal subarachnoid hemorrhage. J Neurosurg 2004;101:408-16

6. Farrell B, Godwin J, Richards S, et al. The United Kingdom transient ischaemic attack (UK-TIA) aspirin trial: final results. J Neurol Neurosurg Psychiatry 1991;54:1044-54

7. Willinsky RA, Peltz J, da Costa L, et al. Clinical and angiographic follow-up of ruptured intracranial aneurysms treated with endovascular embolization. AJNR Am J Neuroradiol 2009;30:1035-40

8. Kang DH, Kim YS, Baik SK, et al. Acute serious rebleeding after angiographically successful coil embolization of ruptured cerebral aneurysms. Acta Neurochir 2010;152:771-81

9. Jartti P, Isokangas JM, Karttunen A, et al. Early rebleeding after coiling of ruptured intracranial aneurysms. Acta Radiol 2010;51: 1043-49

10. Dmytriw AA, Pickett GE, Shankar JJ. Rupture of aneurysms in the immediate post-coiling period. J Neurointerv Surg 2014;6:16-18

11. Egashira $Y$, Yoshimura S, Enomoto $Y$, et al. Ultra-early endovascular embolization of ruptured cerebral aneurysm and the increased risk of hematoma growth unrelated to aneurysmal rebleeding. J Neurosurg 2013;118:1003-08

12. Klompenhouwer EG, Dings JT, van Oostenbrugge RJ, et al. Singlecenter experience of surgical and endovascular treatment of ruptured intracranial aneurysms. AJNR Am J Neuroradiol 2011;32:570-75

13. Güresir E, Beck J, Vatter H, et al. Subarachnoid hemorrhage and intracerebral hematoma: incidence, prognostic factors, and outcome. Neurosurgery 2008;63:1088-93, discussion 1093-94

14. Nagasaka T, Tsugeno M, Ikeda $\mathrm{H}$, et al. Early recovery and better evacuation rate in neuroendoscopic surgery for spontaneous intracerebral hemorrhage using a multifunctional cannula: preliminary study in comparison with craniotomy. J Stroke Cerebrovasc Dis 2011;20:208-13

15. Y Nievas MC, Toktamis S, Haas E, et al. Benefits of adapting minimal invasive techniques to selected patients with spontaneous supratentorial intracerebral hematomas. Neurol Res 2005;27:755-61

16. Ryu CW, Lee CY, Koh JS, et al. Vascular perforation during coil embolization of an intracranial aneurysm: the incidence, mechanism, and clinical outcome. Neurointervention 2011;6:17-22

17. Zang $\mathrm{P}$, Liang $\mathrm{C}$, Shi Q, et al. Intraprocedural cerebral aneurysm rupture during endovascular coiling. Neurol India 2011;59:369-72

18. Kwon BJ, Chang HW, Youn SW, et al. Intracranial aneurysm perforation during endosaccular coiling: impact on clinical outcome, initial occlusion, and recanalization rates. Neurosurgery 2008;63: 676-82, discussion 682-83

19. Naidech AM, Jovanovic B, Liebling S, et al. Reduced platelet activity is associated with early clot growth and worse 3-month outcome after intracerebral hemorrhage. Stroke 2009;40:2398-401

20. Flibotte JJ, Hagan N, O'Donnell J, et al. Warfarin, hematoma expansion, and outcome of intracerebral hemorrhage. Neurology 2004;63: 1059-64 